\title{
Consideraciones del CAVEI para la promoción de la adherencia a la vacunación
}

\author{
Adiela Saldaña ${ }^{1,2}$, Solange Santillana ${ }^{1,3}$, M. Teresa Valenzuela", Jeannette Dabanch ${ }^{1,5}$, \\ Cecilia González ${ }^{6}$, Jaime Cerda7, Johanna Acevedo ${ }^{8}$, Mario Calvo9, \\ Eduardo Díaz ${ }^{10}$, María L. Endeiza ${ }^{11}$, Jaime Inostroza ${ }^{12}$ y Jaime Rodríguez ${ }^{13}$
}

\section{CAVEI considerations for the promotion of vaccination adherence}

\section{Introducción}

\section{T} a vacunación es considerada uno de los mayores logros de la salud pública del siglo XX, reconociéndose ampliamente su utilidad y beneficio. Se le estima como una de las herramientas más costo-efectiva para disminuir la tasa de las enfermedades inmunoprevenibles, lo que ha sido demostrado tras la implementación de campañas y programas de inmunización que han permitido reducir de manera significativa la morbilidad, discapacidad y mortalidad de la población a causa de la enfermedades prevenibles a través de la vacunación ${ }^{1-6}$. Sin embargo, en los últimos años han aumentado los cuestionamientos, la información falsa, el escepticismo, y la desconfianza en cuanto a la utilidad, seguridad y efectividad de las vacunas, convirtiéndose en un desafío global para todos los programas de inmunización, con una posible repercusión nacional ${ }^{7-10}$.

El impacto negativo de la disminución de la adherencia a la vacunación se evidencia con claridad en el continente europeo, con un brote de sarampión; en el período comprendido entre el $1^{\circ}$ de mayo de 2018 y el 30 de abril de 2019 se han notificado 11.149 casos de sarampión, de los cuales 6.636 (59\%) no estaban vacunados, existiendo 13 muertes atribuibles a la afección en este período ${ }^{11}$.

Tanto el contexto social, como los factores educacionales, culturales, socio-económicos, religiosos y creencias, entre otros, impactan en la adherencia a la vacunación y, por ende, en la prevención de enfermedades. El Plan de Acción Global en Vacunas 2012-2020, de la OMS (Global Vaccine Action Plan -GVAP-) incluye dentro de sus propuestas, la incorporación de indicadores para medir la adherencia a la vacunación, a modo de evidenciar los determinantes sociales que influyen en esta adherencia en el ámbito nacional como internacional, así como las medidas implementadas que contribuyen a revertir el fenómeno de falta de adherencia $^{12,13}$.

\section{Antecedentes internacionales y nacionales}

\section{Contexto internacional}

La falta de adherencia a la vacunación como una consecuencia de determinantes de la esfera social, en el escenario internacional se ha traducido, en los últimos años, en un aumento del número de enfermedades prevenibles a través de la inmunización, y siendo un caso característico el brote de sarampión en Europa. Es así como el año 2017 se notificaron 14.451 casos al sistema de vigilancia europeo por 30 países de la Unión Europea, cantidad de casos que fue tres veces superior a las del año 2016 (4.643 casos), brote que se ha mantenido en el continente europeo. Es así como desde el $1^{\circ}$ de enero al 31 mayo del año 2019 se han presentado 8.358 casos $^{11}$.

Esta falta de adherencia a la vacunación ha sido estudiada por expertos y autoridades internacionales, siendo denominada con el término Vaccine Hesitancy, que en español podríamos traducir como reticencia a las vacunas, lo que se define como el retraso en la aceptación o el rechazo a la vacunación a pesar de la disponibilidad de los servicios para acceder a la vacunación. La reticencia a la vacunación es compleja y específica a un contexto, variando a través del tiempo, lugar y la vacuna en particular; se describe, además, como un comportamiento influenciado por una serie de factores, incluyendo los problemas relacionados con la confianza (nivel de confianza en la vacuna, o proveedor), complacencia (el usuario no percibe la necesidad de utilizar vacuna, no valora su utilización), y conveniencia (factores que afectan el acceso a la vacunación $)^{9,10,14}$.

Cuando se habla de personas reticentes a las vacunas se debe tener presente que éstas pertenecen a un grupo heterogéneo en cuanto a grados de indecisión sobre la vacunación, tanto en su concepto general o en relación a vacunas específicas. Hay casos en que existe aceptación de las vacunas, pero con preocupación en ciertos aspectos, otros en los cuales se rechaza o retrasa la administración sólo de vacunas específicas y un tercer grupo, más radical,
${ }^{1}$ Consejo Asesor de Vacunas e Inmunización, Ministerio de Salud de Chile.

${ }^{2}$ Instituto de Salud Pública de Chile.

${ }^{3}$ Servicio de Salud Valparaíso y San Antonio.

${ }^{4}$ Universidad de los Andes ${ }^{5}$ Hospital Clínico Universidad de Chile.

${ }^{6}$ Departamento de

Inmunizaciones, Ministerio de Salud.

${ }^{7}$ Pontificia Universidad Católica de Chile.

${ }^{8}$ Departamento de Epidemiología,

Ministerio de Salud.

${ }^{9}$ Facultad de Medicina,

Universidad Austral, Valdivia,

Chile.

${ }^{10} \mathrm{Facultad}$ de Medicina,

Universidad de Chile.

"Clínica Universidad de Los

Andes.

${ }^{12}$ Universidad de La Frontera,

Temuco, Chile.

${ }^{13}$ Clínica Alemana, Santiago,

Chile.

Recibido: 20 de agosto de 2019 (versión final)

Correspondencia a: Jeannette Dabanch Peña jeannettedabanch@gmail.com

Magdalena Bastías García caveichile@gmail.com 
que rechaza todas las vacunas ${ }^{6}$. La reticencia en la vacunación es un proceso continuo que podría ir desde una alta demanda hasta el rechazo total de las vacunas, vale decir, desde una persona quien exige que se le administre las vacunas disponibles, hasta quien duda o rechaza definitivamente administrarse las vacunas disponibles en el Programa Nacional de Inmunización (PNI) ${ }^{9}$.

En relación a la reticencia a la vacunación el Grupo Consultivo Estratégico de Expertos de OMS (Strategic Advisory Group of Experts on Immunization -SAGE), evaluó una serie de modelos conceptuales para comprender y agrupar los determinantes que la afectan, siendo estos identificados como: Confianza, Complacencia y Conveniencia9.

La confianza fue definida como la existencia de una convicción, en la efectividad y seguridad de las vacunas, confianza en el sistema que las proporciona, incluyendo la confiabilidad y la competencia de los servicios de

\section{Tabla 1. Determinantes de la reticencia a la vacunación}

$\begin{array}{ll}\text { Influencias } & \text { Comunicación y medios } \\ \text { del contexto } & \text { Líderes de opinión, encargados de programas de inmunización, lobby anti } \\ & \text { y pro vacunación } \\ \text { Influencias históricas } & \text { Religión, cultura, género, factores socio-económicos } \\ & \text { Políticas, regulaciones } \\ & \text { Barreras geográficas } \\ & \text { Percepción de la industria farmacéutica }\end{array}$

Influencias individuales y de grupo

Personal, familia y miembros de la comunidad, experiencia con vacunaciones anteriores, incluyendo dolor

Creencias, actitudes acerca de la salud y de la prevención

Nivel de conocimiento y conciencia que se tenga en relación a las vacunas

Sistema de salud, confianza y experiencia personal

Riesgo/beneficio (percepción, heurística)

Inmunización como una norma social vs falta de necesidad y daño

\section{Problemas Riesgo/beneficio (evidencia epidemiológica y científica)}

específicos sobre

vacunas y

vacunación

Introducción a nuevas vacunas o nueva formulación o a nueva recomendación por una vacuna existente

Formas de administración

Diseño del programa de vacunación, forma de distribución (p.ej. programa rutinario o campaña de vacunación)

Confiabilidad y/o fuente de suministro de vacuna y/o equipo de vacunación

Calendario de vacunación

Costos

Fuerza de la evidencia científica en la recomendación y/o base del conocimiento y/o actitud de los profesionales de la salud

Adaptado de reporte de trabajo de SAGE en reticencia de vacunas 01 oct $2014^{9}$ salud y sus profesionales, así como la confianza en las motivaciones de los responsables de las políticas públicas que deciden sobre cuáles son las vacunas necesarias para un programa de inmunización ${ }^{9,10}$.

La complacencia en la vacunación existe cuando se percibe que las enfermedades prevenibles por vacunas poseen un bajo riesgo para la salud, además se considera la vacunación como una medida de prevención innecesaria. El éxito de un programa de inmunización puede, paradójicamente, dar como resultado la complacencia, $\mathrm{y}$, en última instancia, la vacilación, ya que las personas evalúan los riesgos de las vacunas contra los riesgos de enfermedades que ya no son comunes ${ }^{9,10}$.

La conveniencia de la vacuna es medida por la disponibilidad física de ella, asequibilidad y disposición a pagar, accesibilidad geográfica, capacidad de comprensión (lenguaje y alfabetización en salud) y disponer de los servicios de inmunización. Otros factores que afectan la decisión de vacunarse y podrían llevar a la reticencia son la calidad del servicio de vacunación (real o percibido) y el grado de implementación en un momento, lugar y contexto cultural que sea conveniente y cómodo para la población ${ }^{10}$.

Asimismo, el SAGE realizó una matriz más compleja en la cual se identificaron determinantes que influyen en la reticencia a la vacunación y que clasificaron en tres grupos: influencia de contexto, influencia de individuos y de grupo y, finalmente, problemas específicos sobre vacunas y vacunación, los que se detallan en la Tabla 1.

La reticencia a las vacunas es una preocupación permanente para distintas organizaciones y expertos en materia de vacunas; es así como el GVAP incorporó dentro de los objetivos estratégicos 2011-2020, indicadores que permiten evaluar el nivel de confianza en la vacunación y el SAGE recomendó a los programas de inmunización incorporar nuevos indicadores para identificar las principales razones del rechazo a la vacunación, identificar si la respuesta obtenida es una opinión o está basada en evidencia; conocer si en los últimos cinco años se dispone de alguna evaluación, acerca del grado de confianza de la población con respecto a la vacunación a nivel nacional o local ${ }^{12,13,15,16}$.

Por otra parte, de acuerdo al Centro Europeo para la Prevención y Control de Enfermedades (ECDC), los principales determinantes de reticencia a vacunas en Europa son las dudas en relación a la seguridad de vacunas, falta de información, percepción de bajo riesgo de enfermedades graves, y percepción que las vacunas no son efectivas ${ }^{8}$.

GVAP publicó las principales causas a la reticencia de la vacunación en la Región de las Américas durante el período 2014-2016. A continuación, se presentan los determinantes principales para la reticencia a la vacunación en la Tabla $2^{15,16}$. 


\begin{tabular}{|c|c|c|c|c|c|}
\hline 2014 & & 2015 & & 2016 & \\
\hline Determinante & Frecuencia & Determinante & Frecuencia & Determinante & Frecuencia \\
\hline $\begin{array}{l}\text { Religión, cultura, género, } \\
\text { socio-económico }\end{array}$ & 11 & $\begin{array}{l}\text { Relación riesgo/beneficio de } \\
\text { las vacunas }\end{array}$ & 16 & $\begin{array}{l}\text { Religión, cultura, género, } \\
\text { socio-económico }\end{array}$ & 15 \\
\hline $\begin{array}{l}\text { Relación riesgo/beneficio } \\
\text { de las vacunas }\end{array}$ & 11 & $\begin{array}{l}\text { Líderes de opinión, lobbies } \\
\text { de grupos anti-vacunas }\end{array}$ & 11 & $\begin{array}{l}\text { Relación riesgo/beneficio de } \\
\text { las vacunas }\end{array}$ & 15 \\
\hline Comunicación y medios & 6 & $\begin{array}{l}\text { Religión, cultura, género, } \\
\text { socio-económico }\end{array}$ & 9 & Comunicación y medios & 8 \\
\hline
\end{tabular}

\section{Contexto nacional}

\section{Coberturas de vacunación en Chile}

De acuerdo a datos entregados por el Departamento de Inmunizaciones del MINSAL, las vacunas que presentan las coberturas más bajas en Chile en el período 2010-2017 son:

- Vacuna neumocócica polisacárida, cuya población objetivo son las personas mayores de 65 años de edad, en dosis única, con una cobertura promedio de $22 \%$, y una tendencia a la disminución gradual desde el año 2014, donde se logró una cobertura de 34,7\% y llegando a 14,2\% el año 2017.

- Vacunación contra el virus del papiloma humano (VPH), con un promedio de $83,2 \%$ para el período 2014-2017, siendo la cifra más baja el año 2016 con $77,9 \%$ y recuperándose el año 2017 con $83 \%$. Respecto de la inmunización contra el VPH, el año 2017 se vacunaron pacientes que no fueron vacunadas durante el año 2016, a pesar que les correspondía ser vacunadas por calendario de inmunización, registrándose así un total de 8.760 primeras dosis de vacuna administrada en niñas de $5^{\circ}$ básico, evidenciándose un avance en la actualización de los esquemas de vacunación en todas las regiones del país, cuyas causas de desactualización del esquema vacunal aún no están especificadas, pero podrían incluir casos de rechazo a la vacunación. Similar situación se observó en $6^{\circ}$, $7^{\circ}$ y $8^{\circ}$ básico, donde los datos de registro muestran 683 primeras dosis en total durante el año 2017, esto muestra que el rechazo de vacunación fue temporal en los grupos de $5^{\circ}, 6^{\circ}, 7^{\circ}$ y $8^{\circ}$ básico que se vacunaron posteriormente ${ }^{17}$.

Los demás datos de coberturas de las vacunas que integran el Programa de Nacional de Inmunización en Chile entre los años 2010 y 2018 son presentados en la Tabla $3^{17}$.
Cabe señalar que las estimaciones de coberturas pueden verse afectadas por el uso de distintos denominadores poblacionales, que pueden generar subestimación o sobreestimación de la población real ${ }^{18-20}$.

Hasta el año 2010, las coberturas de vacunación eran calculadas utilizando población obtenida a través del método Taucher, mientras que, a partir de 2011, utilizando Población INE ${ }^{21}$. Desde el 2017 se utiliza como denominador el grupo menor de 2 años, la población de Recién Nacidos Inscritos en el Registro Civil ${ }^{22}$. En el caso particular de la cobertura de la vacuna anti-neumocócica polisacárica 23 valente se usa como denominador la población de 65 años INE 2013-2020.

\section{Rechazo de la vacunación en Chile}

A partir del año 2010, Chile cuenta con un repositorio nacional de vacunas administradas, sistema conocido como Registro Nacional de Inmunizaciones (RNI), oficializado a partir del año $2013^{23,24}$. El RNI permite la trazabilidad de cada individuo vacunado, mediante el RUT y cada producto administrado y de otras variables, como el rechazo a la vacunación ${ }^{25}$. De esta forma, el sistema es capaz de identificar los casos en que los padres, cuidadores o individuos, rechazan la vacunación (Figura 1 y Tabla 4). Este registro se encuentra disponible en cada centro de salud público y privado en convenio para vacunas pertenecientes al PNI. A la fecha, el mayor rechazo a la vacunación lo presenta la vacuna VPH con 7\% el año 2017 (Figura 1).

Una limitación del RNI es que no especifica los motivos del rechazo a la vacunación, por lo que con estos datos no es posible conocer en detalle cuáles son los determinantes del rechazo en Chile.

Por otro lado, el sistema tampoco permite identificar los casos de duda o vacilación en torno a la vacunación, la que, según otras fuentes, estaría relacionada con aspectos 


\begin{tabular}{|c|c|c|c|c|c|c|c|c|c|c|}
\hline \multirow[t]{2}{*}{ Vacuna } & \multirow[t]{2}{*}{ Dosis } & \multicolumn{9}{|c|}{ Cobertura (\%) } \\
\hline & & 2010 & 2011 & 2012 & 2013 & 2014 & 2015 & 2016 & 2017 & 2018 \\
\hline $\mathrm{BCG}^{5}$ & RN & 95,9 & 94,4 & 93,3 & 91,9 & 96,1 & 93,9 & 91,1 & 96,2 & 100 \\
\hline Pentavalente (incluye hexavalente) & $3^{\circ}$ dosis & 92,6 & 94,8 & 92,8 & 86,9 & 92,9 & 96,6 & 90,7 & 92,1 & 94,9 \\
\hline Neumocócica conjugada & $1^{\circ}$ refuerzo & & & 83,3 & 84,7 & 89,3 & 89,9 & 92,7 & 88,2 & 93,6 \\
\hline SRP & única & 93,5 & 92,0 & 90,6 & 89,7 & 93,9 & 88,5 & 98,6 & 88,2 & 93,3 \\
\hline Meningocócica (vacuna tetravalente ACYW-135) & única & & & & & 73,7 & 94,3 & 94,3 & 89,1 & 92,1 \\
\hline Polio oral (incluye hexavalente) & $1^{\circ}$ refuerzo & 89,1 & 91,2 & 90,8 & 85,3 & 87,0 & 90,3 & 90,8 & 82,0 & 87,2 \\
\hline SRP & $1^{\circ}$ refuerzo & 83,8 & 78,7 & 74,7 & 75,6 & 87,5 & 89,0 & 88,1 & 90,2 & 93,1 \\
\hline dTpa & $1^{\circ}$ refuerzo & & & & 83,8 & 88,2 & 88,0 & 88,3 & 100 & 92,5 \\
\hline $\mathrm{VPH}^{*}$ & $4^{\circ}$ básico & & & & & 86,4 & 85,8 & 77,9 & 83,0 & 87,2 \\
\hline Neumocócica adulto & única & & 18,9 & 25,6 & 20,8 & 34,7 & 23,3 & 16,5 & 14,2 & 33,2 \\
\hline \multicolumn{11}{|c|}{$\begin{array}{l}\text { Fuente: Coberturas 2010-2017. Departamento de Inmunizaciones, MINSAL. V. Torres [Comunicación personal]. } 5 \text { de marzo de 2018. Coberturas de vacunación progra- } \\
\text { mática, reporte vacunas 2018. Departamento de Estadísticas e Información de Salud. MINSAL. Disponible en: http://www. deis.cl/estadisticas-inmunizaciones/. } 15 \text { de julio } \\
\text { 2019. sRecién nacidos vivos año 2017, con residencia conocida, primeros seis meses de la cohorte anual. Para el año 2018, se considera el total de Recién Nacidos Vivos } \\
\text { del año 2018. "Se incorporó el año } 2014 \text { como vacuna programática. }{ }^{*} \text { Matrículas MINEDUC niñas } 4^{\circ} \text { básico. }\end{array}$} \\
\hline
\end{tabular}

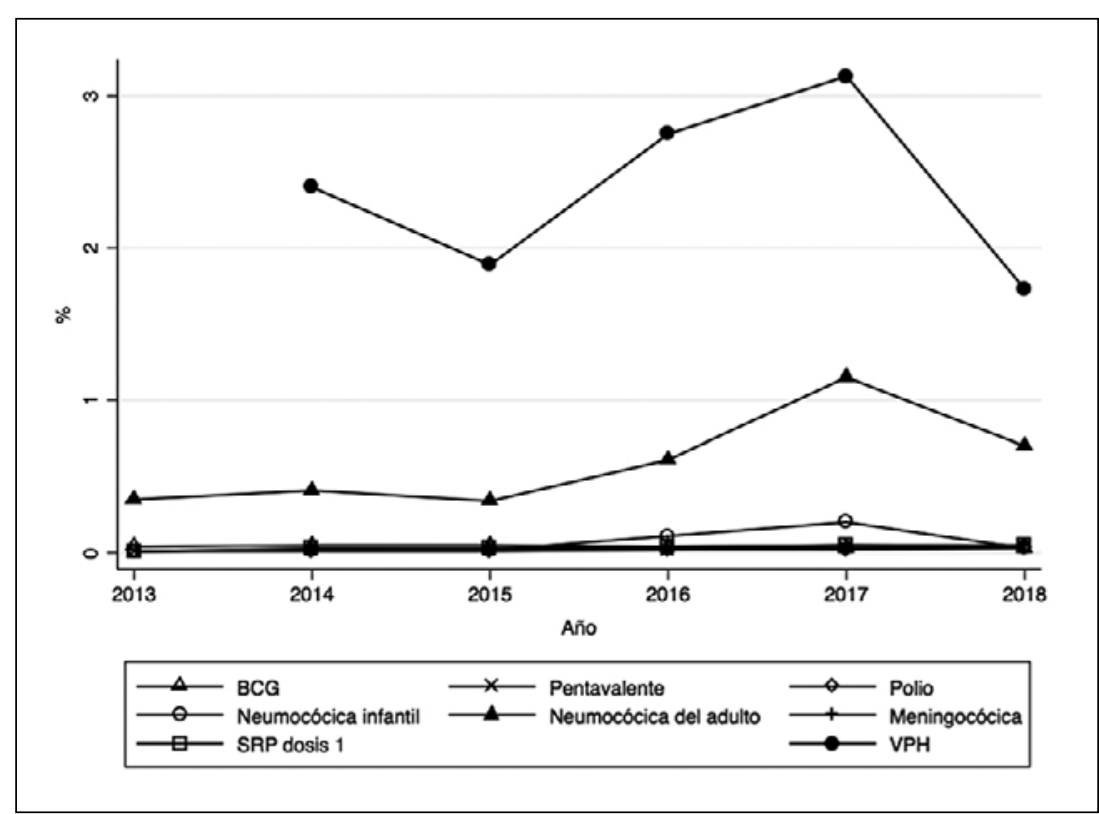

Figura 1. Rechazo a vacunas en Chile, años 2013-2018. Fuente: elaboración propia, datos sistema RNI, MINSAL al 15 de julio de 2019. VPH considera vacunación hasta $8^{\circ}$ básico y todas las dosis. Porcentaje de rechazo: rechazos dividido por los rechazos más las dosis administradas de vacunas.

comunicacionales y educativos ${ }^{26,27}$. Otro sesgo, que se estima es un porcentaje menor, son los vacunatorios o salas de procedimiento, que no están en convenio con el MINSAL; éstos no poseen obligatoriedad de usar RNI, y aunque el MINSAL ha generado la posibilidad de establecer un convenio especial para que tengan acceso al registro al RNI no todos ellos lo utilizan. Estudios muestran que los padres coinciden en la importancia de contar con información para tomar decisiones en torno a la vacunación y que la información existente no es suficiente para aclarar las dudas ${ }^{26}$.

El desglose anual del número de personas vacunadas por cada rechazo según tipo de vacuna se presenta en la Tabla 4. Para el caso de vacuna VPH, se aprecia que por cada 14 niñas vacunadas el año 2017 existe 1 caso de rechazo.

Por otra parte, la "Encuesta bimestral de monitoreo de indicadores y percepción del Sistema de Salud Chileno" realizada por el Instituto de Políticas Públicas en Salud de la Universidad San Sebastián del año 2016, que entrevistó a 1.003 personas, señala que $5 \%$ de las personas declaró no vacunar a sus hijos, $5 \%$ creía que "las vacunas eran perjudiciales para su hijo" y $21 \%$ que "algunas veces eran perjudiciales". Cuando se les consultó acerca de cuál debería ser el rol del Estado cuando los padres deciden no vacunar, tanto padres que sí vacunaban a sus hijos como los que no lo hacían, respondieron que el Estado "debía educar", 74 y $68 \%$, respectivamente. Ante la consulta acerca de si cree que lo padres que no vacunan a sus hijos debieran recibir una multa por no realizarlo, 20\% de los padres que vacunan a sus hijos opinó que deberían recibir una multa y $12 \%$ consideró que se le deben cancelar los beneficios sociales a esos padres ${ }^{28}$. 
Signos de rechazo y dudas que pueden afectar la adherencia a la vacunación en Chile

\section{Recursos de Protección*}

Un signo del rechazo a la vacunación en Chile han sido los recursos de protección interpuestos por padres en relación a la vacunación de sus hijos. El año 2016 se presentaron 14 causas en las cortes chilenas contra la vacunación de vacuna VPH en 11 ciudades y ninguna de ellas fue acogida. No obstante, ese año (2016) la cobertura de vacunación para VPH fue la más baja, de $77,9 \%$, en comparación a otros años.

\section{Redes sociales con mensajes en contra de las vacunas}

Actualmente, es común encontrar en redes sociales como Facebook mensajes en contra de alguna vacuna en particular y/o que hacen uso de mitos como verdades. Estos mensajes con información falsa y sin base cientíica, pueden influir el juicio de las personas en relación a las vacunas. Ejemplos de grupos que emiten estas comunicaciones son "Libertad de vacunación para un Chile democrático" que posee 11.564 miembros, creado $2013^{29}$ y el grupo "No a Gardasil" con 13.121 miembros, creado el año 2015, los que poseen claros mensajes en contra de la vacunación que son vistos permanentemente por un número creciente de personas ${ }^{30}$. Por otro lado, este fenómeno deja en evidencia una contraparte que presenta una carencia de información validada que los oriente y eduque en materia de beneficio y seguridad de las vacunas, en un formato amigable, oportuno, en plataformas electrónicas y medios comunicacionales de fácil acceso a la población y profesionales de salud.

Dados estos antecedentes, el CAVEI extiende las siguientes consideraciones para promover la adherencia a la vacunación:

\begin{tabular}{|l|}
\hline Dimensión estructural \\
\hline - Implementar un sistema organizacional o complementar los ya \\
existentes, de modo que se permita abordar la temática de ad- \\
herencia, rechazo y reticencia, medirla, evaluarla e implementar \\
estrategias específicas en torno a la vacunación en forma perma- \\
nente. \\
- Crear comités multidisciplinarios que evalúen las coberturas de \\
vacunación semestralmente, que aborden, en conjunto con el \\
Departamento de Inmunizaciones, las dificultades de lograr \\
altas coberturas en lactantes, escolares, adulto mayor, mujeres \\
embarazadas y campañas.
\end{tabular}

* S. Abarca [Comunicación personal]. 31 de marzo de 2017, Información enviada por correo electrónico desde la coordinadora unidad judicial MINSAL.
Tabla 4. Número de personas vacunadas antes de que se produzca un rechazo de vacuna por solicitud de persona, 2013-2018

\begin{tabular}{lrrrrrr}
\hline Vacuna programática & \multicolumn{6}{c}{ Número de vacunados por cada rechazo } \\
& $\mathbf{2 0 1 3}$ & $\mathbf{2 0 1 4}$ & $\mathbf{2 0 1 5}$ & $\mathbf{2 0 1 6}$ & $\mathbf{2 0 1 7}$ & $\mathbf{2 0 1 8}$ \\
\hline BCG & 2.812 & 1.836 & 2.043 & 2.605 & 3.157 & 3.226 \\
Pentavalente & 7.667 & 4.103 & 6.136 & 4.225 & 3.369 & 3.221 \\
\hline Polio & 11.463 & 7.636 & 11.270 & 6.044 & 4.126 & 3.353 \\
Neumocócica infantil & 8.993 & 5.759 & 4.587 & 874 & 499 & 3.631 \\
Neumocócica adulto & 288 & 244 & 295 & 162 & 86 & 141 \\
\hline Meningocócica & & 3.137 & 4.000 & 2.759 & 2.070 & 2.418 \\
SRP 1d & 9.226 & 3.061 & 3.002 & 2.468 & 1874 & 2.076 \\
VPH &. & 41 & 52 & 35 & 31 & 57 \\
Total & 4.476 & 988 & 431 & 296 & 340 & 455 \\
\hline
\end{tabular}

Fuente: Departamento de Inmunizaciones, MINSAL. Datos al 15 de julio de 2019. Rechazos por solicitud de la persona.

- Estudiar la forma funcional, legal y financiera para establecer un sistema de compensación de Evento Supuestamente Atribuido a Vacunación o Inmunización- ESAVI, en Chile.

- Generar la coordinación permanente (mensual) entre el PNI y Ministerio de Educación-MINEDUC: incorporar a un representante del MINEDUC en reuniones del CAVEI, a modo que se mejore la comunicación y coordinación entre MINEDUC-MINSAL, Programas de salud del adulto mayor, crónicos y de salud reproductiva. - Integrar los componentes del Programa Nacional de Inmunizaciones-PNI en forma transversal a las otras actividades que se realizan en la Atención Primaria de Salud (salud reproductiva, salud del niño, alimentación complementaria, salud de la mujer, etc.).

\section{Dimensión de recursos humanos}

- Fortalecer el recurso humano que actualmente lidera el PNI en los establecimientos públicos y privados en convenio, a través de un programa de capacitación continua y exclusividad en las labores de gestión y ejecución del mismo.

- Incorporar al trabajo del PNI a sociólogos y/o psicólogos y/o comunicadores a modo de que mejoren las estrategias y herramientas para abordar problemas de adherencia.

- Capacitar a los equipos de salud con al menos un taller anual con enfoque hacia el reforzamiento de las normas técnicas, de los registros y actualizaciones sobre los avances de los programas de inmunización

\section{Dimensión de comunicación social}

- Implementar un Plan de Comunicación Social proactivo para el programa regular, que permita posicionar la temática de la vacunación, beneficio y seguridad de las vacunas en la comunidad, con énfasis del plan en los grupos de población con menor adherencia.

- Planificar educación en "terreno" a través de cursos regionales que incorporen a personal local, PNI central, CAVEI, CCI SOCHINF, Sociedad Chilena de Pediatría, Sociedad Chilena de Ginecología y Obstetricia y otras. 


\begin{tabular}{l} 
- Realizar actividades comunicacionales masivas en la Semana de \\
la Vacunación en las Américas, que posicionen los beneficios de \\
la vacunación, con la presencia de las más altas autoridades de \\
salud, políticos, sociedades científicas y otros actores. \\
\hline Dimensión de comunicación social \\
\hline - Formación de líderes en salud quienes a su vez repliquen y difun- \\
dan conocimientos en la población \\
- Crear la figura de "Community Manager" que conduzca redes \\
sociales formales, difundiendo pro-activamente información acer- \\
ca de las vacunas como: los beneficios de las vacunas, inicio de \\
campañas, duración de ellas, resultados de coberturas, preguntas \\
y respuestas. \\
- Difundir la información de coberturas a la comunidad científica \\
y de la sociedad en su conjunto. \\
- Fortalecer el repositorio de la información actualmente existente \\
en la web del MINSAL en cuanto a: presentación amigable para \\
la comunidad, de libre acceso, conocida por todos \\
- Entrega de material educativo orientado a padres y población es- \\
colar durante las instancias de vacunación escolar que contempla \\
el PNI. \\
Dimensión de docencia y desarrollo de proyectos \\
\hline - Incorporar en la malla curricular de todas las escuelas de salud \\
módulos sobre vacunas: los jóvenes son más capaces de influir \\
en creencias y comportamiento de futuras generaciones \\
- Realizar trabajos colaborativos entre academia, sociedades cien- \\
tíficas, MINSAL, ISP en el fortalecimiento de la adherencia, en el \\
que se incluya diagnóstico de la situación en Chile y acciones que \\
la mejoren.
\end{tabular}

Generar un curso que se dicte cada dos años multidisciplinario, común y obligatorio para becarios de infectología adultos y pediátricos, inmunólogos, salubristas y electivo para bioquímicos/ sociólogos/enfermeras/miembros de la industria farmacéutica u otras profesiones que se dediquen al tema de vacunas, donde se capacite globalmente en temas desde conceptos generales, específicos, éticos, prácticos y manejo de medios, para generar masa crítica con conocimiento adecuado para desarrollar planes a futuro e integrar comités asesores

\section{Dimensión de sistemas de información}

- Aplicar instrumentos de diagnóstico para conocer cuáles son los motivos de las dudas en vacunas, que incluya el componente regional y comunal, en particular en vacunación contra VPH y vacuna neumocócica adulto, que presentan el mayor rechazo. - Implementar mejoras en el actual Registro Nacional de Inmunizaciones (RNI): a) incorporar campos específicos que permitan pesquisar cuáles son los motivos de rechazo de la vacunación b) incorporar el campo de registro específico acerca de si la persona presenta algún grado de reticencia a la vacunación, especificar las razones, evaluarlas periódicamente, desarrollar acciones específicas para ellas.

- Incorporar indicadores que permitan monitorear el nivel de adherencia de la vacunación escolar

Nuestros agradecimientos a Magdalena Bastias García, secretaria técnica del CAVEI por Coordinar la obtención de datos, facilitarnos material y la revisión general del manuscrito.

\section{Referencias bibliográficas}

1.- Organización Panamericana de la Salud. 2018. El Programa de Inmunización en las Américas: su historia contada a través del Boletín de Inmunización. Disponible en http://www.paho.org/inb/index. php?option $=$ com_content $\&$ view $=$ article $\&$ id $=13$ $\&$ Itemid $=46 \&$ lang $=$ es [citado el 25 de febrero de 2018].

2.- Organización Mundial de la Salud, Fondo de las Naciones Unidas para la Infancia, Banco Mundial. Vacunas e inmunización: situación mundial, tercera edición. Ginebra, Organización Mundial de la Salud, 2010. Disponible en http://whqlibdoc.who.int/ publications/2010/9789243563862_spa.pdf

3.- Andrus J K, Bandyopadhyay A S, Danovaro Holliday M C, Dietz V, Domingues C, Figueroa $\mathrm{J}$ P, et al. El pasado, el presente y el futuro de la inmunización en las Américas. Rev Panam Salud Publica 2017, 41: 1-5. https://docplayer. es/77238811.

4.- Etienne C F. Expanded Program on Immunization in the Americas: 40 years. Rev Panam Salud Publica. 2017;41: e139. doi: 10.26633/RPSP.2017.139.

5.- Leal F, López P. Vacunas en Pediatría.
Tercera edición. Editorial Médica Internacional, 2008. Disponible en http:// books.google.cl/books?id=2NKXhQ_ oo6UC\&printsec=frontcover\&sour ce $=$ gbs_ge summary_r\&cad $=0 \# v=$ onepage $\& q \& \mathrm{f}=$ false .

6.- Evans R. Diez logros sanitarios más importantes en el siglo veinte. Vol.24, N.1.2001. Disponible en http://www. scielo.org.ve/scielo.php?pid=S079804692001000100003\&script=sci_arttext.

7.- Karafillakis E A, Larson H J. The benefit of the doubt or doubts over benefits? A systematic literature review of perceived risks of vaccines in European populations. Vaccine 2017; 35: 4840-50. doi: 10.1016/j. vaccine.2017.07.061.

8.- European Centre for Disease Prevention and Control. Catalogue of interventions addressing vaccine hesitancy. Stockholm: ECDC; 2017.

9.- World Health Organization (WHO). (2014). Report of the SAGE working group on vaccine hesitancy. Geneva, Switzerland: WHO. Disponible en http://www.who.int/ immunization/sage/meetings/2014/october/1_ Report_WORKING_GROUP_vaccine hesitancy_final.pdf [citado el $\overline{2} 5$ de febrero de 2018].
10.- MacDonald N E. Vaccine hesitancy: Definition, scope and determinants. Vaccine 2015; 33 (34): 4161-4. doi: 10.1016/j. vaccine.2015.04.036.

11.- Sitio web Centro Europeo de Control y prevención de Enfermedades (ECDC). Measles cases in the EU treble in 2017, outbreaks still ongoing. Disponible en https://ecdc.europa.eu/ en/news-events/measles-cases-eu-treble-2017outbreaks-still-ongoing [citado el 25 de febrero de 2018].

12.- Sitio web Organización Mundial de la Salud (OMS). Determinantes en Salud. Disponible en http://www.paho.org/hq/ index.php?option $=$ com_content\&view $=\mathrm{a}$ rticle\&id=5165\%3A2011-determinantshealth\&catid $=5075 \% 3$ Ahealthpromotion\&Itemid=3745\&lang=es [citado el 25 de febrero de 2018].

13.- Sitio web Organización Mundial de la Salud (OMS). Global Vaccine Action Plan Indicator Portal. Disponible en http://apps.who.int/gho/ cabinet/gvap.jsp [citado el 25 de febrero de 2018].

14.- Jarret C, Wilson R. Strategies for adressing vaccine hesitancy. A systematic review. Vaccine 2015; 33: 4180-90. doi: 10.1016/j. vaccine.2015.04.040. 
15.- Sitio web Organización Mundial de la Salud (OMS). Global VaccineAccion Plan. Disponible en http://www.who.int/immunization/global vaccine_action_plan/GVAP_doc_2011_2020/ en/ [citado el 25 de febrero de 2018].

16.- Sitio web Organización Mundial de la Salud (OMS). Global Vaccine Action Plan Monitoring, evaluation, and accountability Secretariat Annual report 2017 http://www.who. int/immunization/global_vaccine_action_plan/ web_gvap_secretariat_report_2017.pdf?ua $=1$ [citado el 25 de febrero de 2018].

17.- Ministerio de Salud de Chile. Departamento de Inmunizaciones. Resumen Informe Vacunación Escolar 2017. Datos al 31-12-2017.

18.- Gobierno de Chile. Ministerio de Salud. Departamento de Estadísticas e Información de Salud. Estimación de la población de 0 a 5 años por grupo de edades programáticas. Documento de uso interno recopilado desde los archivos del Sub Departamento de Administración de Información en Salud perteneciente al Servicio de Salud Valparaíso San Antonio.

19.- BayGuiomar. Estimaciones indirectas de indicadores demográficos para áreas menores: Situación en América Latina.
Disponible en : https://repositorio.cepal.org/ bitstream/handle/11362/12694/NP71-01_ es.pdf? sequence $=1$.

20.- Chackiel J. Los Censos en América Latina: Nuevos Enfoques. Disponible en https://repositorio.cepal.org/bitstream/ handle/11362/12731/np75045072_ es.pdf? sequence $=1 \&$ isAllowed $=\mathrm{y}$.

21.- Rajs D. Ex Jefe Departamento de Estadísticas e Información de Salud, Ministerio de Salud de Chile. Entrevista Personal. Realizada el 31 de mayo de 2013.

22.- Ministerio de Salud. Departamento de Estadísticas e Información en Salud. Informe: Metodología de Monitoreo Cobertura Vacunas Programáticas en menores de 2 años. 2017.

23.- Ministerio de Salud. Departamento de Estadística e Información de Salud. Roles y funciones en el ámbito de sistema de registro nacional de inmunizaciones (RNI). Mayo 2015.

24.- Ministerio de Salud. Ordinario B52 No 4116 del $31 / 12 / 2012$

25.- Ministerio de Salud. Departamento de Estadística e Información de Salud. Manual Registro Nacional de Inmunizaciones: Módulo Vacunas Programáticas. Disponible en http:// www.deis.cl con clave de acceso de gestión interna.

26.- Veliz L, Campos C, Vega P. Conocimiento y actitudes de los padres en relación a la vacunación de sus hijos. Disponible en https:// scielo.conicyt.cl/pdf/rci/v33n1/art05.pdf.

27.- González I, Cuadrado C. Ponencia en Congreso Chileno de Salud Pública. "Decidí no vacunar a mi hijo": Controversias sobre la vacunación en Chile. 2014. Material facilitado por Ingrid González Torres, email de contacto: gringrid6@ gmail.com.

28.- Encuesta bimestral de monitoreo de indicadores y percepción del sistema de salud chileno, IPPS-Universidad San Sebastián. 2016.

29.- Libertad de vacunación para un Chile Democrático. EnfiNet [Perfil en Facebook]. Facebook. 2018. Recuperado a partir de https://www.facebook.com/groups/ Libertaddevacunacion [citado el 27 de febrero de 2018].

30.- No a GardasilEnfiNet [Perfil en Facebook]. Facebook. 2018. Recuperado a partir de https://www.facebook.com/ groups/1476696132637488/ [citado el 27 de febrero de 2018]. 